\title{
Low-Cost Thin and Flexible Screen-Printed Pressure Sensor ${ }^{\dagger}$
}

\author{
Daniel Gräbner 1,*, Marcel Tintelott ${ }^{2}$, Gerrit Dumstorff ${ }^{2}$ and Walter Lang 1,2 \\ 1 Friedrich-Wilhelm-Bessel-Institute Research Society, Bremen, Germany; wlang@imsas.uni-bremen.de \\ 2 Institute for Microsensors, University of Bremen, -Actuators and -Systems, Bremen, Germany; \\ marcel.tintelott@gmx.de (M.T.); gdumstorff@imsas.uni-bremen.de (G.D.) \\ * Correspondence: graebner@fwbi-bremen.de; Tel.: +49-421-218-62575 \\ + Presented at the Eurosensors 2017 Conference, Paris, France, 3-6 September 2017.
}

Published: 11 August 2017

\begin{abstract}
Commercial pressure sensors are often fabricated using well-established silicon micromachining technologies. The thickness and stiffness of silicon-based sensors make them in most cases unsuitable for the integration into materials and surfaces. We present a flexible pressure sensor fabricated by printing technology. Therefore, an intrinsically pressure sensitive ink is screen printed on interdigital electrodes on a thin and flexible foil substrate. The sensor shows sufficient sensitivity and is applicable in a wide pressure range from 0 to $2 \mathrm{MPa}$. The sensor can completely be fabricated using a low-cost screen printing process. It is very thin and flexible, making it possible to be applied on curved surfaces or to be integrated into materials in a minimal invasive way.
\end{abstract}

Keywords: flexible electronics; pressure sensor; screen-printing; surface functionalization; material integrated sensing

\section{Introduction}

Widely used silicon-based pressure sensors were invented almost 50 years ago. Common siliconbased pressure sensors use either the piezoresistive or capacitive sensing principle and are fabricated by well-established silicon micromachining technologies [1]. Even though the lateral dimensions as well as the functionality of the sensors have been improved, the major disadvantages of silicon-based pressure sensing, namely the minimum height and the brittleness, have not yet been worked on. There were a few approaches trying to fabricate thin silicon-based pressure sensors, but they require a costly change in fabrication methods [2].

Low-cost, thin and flexible pressure sensors for the application in a wide pressure range are desired for many applications such as material integrated sensing [3] and surface functionalization [2]. Thin, foil-based screen-printed pressure sensors based on pressure sensitive organic transistors have been reported lately [4]. However, they require a complex printing process as well as suitable electronics for excitation and readout. Resistive, foil-based pressure sensors, so called Force Sensitive Resistors (FSR), have also been investigated, but they usually require a two foil fabrication process, where the electrodes are on one foil and a conducting material on another [5]. Figure 1a shows the cross-sectional view of an FSR sensor. Simple resistive, printed pressure sensors have also been developed, but only for the use at low pressures [6].

We present a low-cost thin and flexible screen-printed resistive pressure sensor for highpressure applications. A render image of the sensor is shown in Figure 1b. The pressure sensitivity is based on a percolation effect within the pressure sensitive layer. The distance between conductive particles in a polymer matrix is lowered by the applied pressure leading to a decrease in resistance of the layer. This can either be caused by reducing the tunneling distance, when tunneling effects are 
mainly responsible for charge transportation, or by simply decreasing contact resistance between the particles [7].

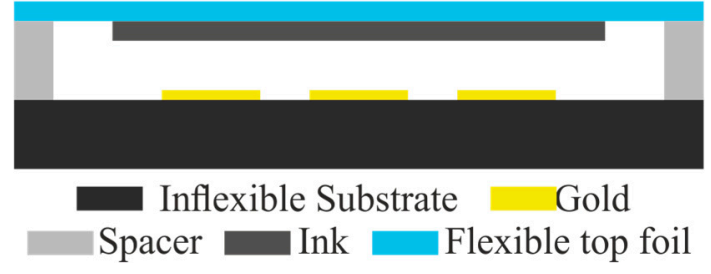

(a)

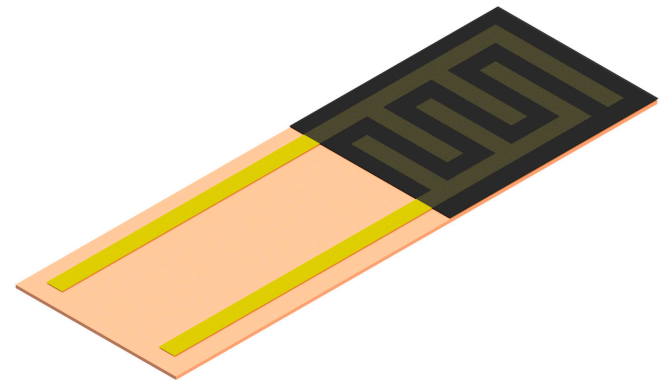

(b)

Figure 1. (a) Cross-sectional view of a Force Sensing Resistor [3]; (b) Render image of presented sensor.

\section{Materials and Methods}

The fabrication steps for the presented sensor are depicted in Figure 2. A $5 \mu \mathrm{m}$ thin and flexible Polyimide foil (U-Varnish S) is fabricated on a handling wafer as seen on Figure 2a. Interdigital electrodes are deposited onto the foil by screen-printing of a conductive silver paste (SunChemical Conductive Silver) with a thickness of approx. $3 \mu \mathrm{m}$ (Figure 2b). The printed silver tracks show a very low and, thus, neglectable resistance. A two-component, conductive, intrinsically pressure sensitive ink (Loctite ECI 7004LR and NCI7002) is then deposited on the electrodes by screen-printing (c).

a)

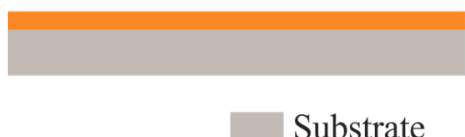

b)

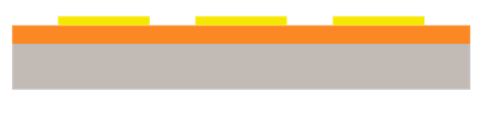

Foil Silver ink c)

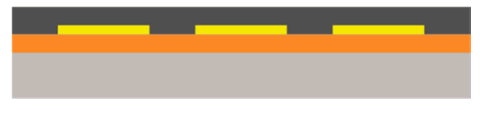

Pressure sensitive ink

Figure 2. Fabrication process of the presented sensor: (a) Fabrication of Polyimide foil on substrate wafer, (b) Deposition and structuring of gold interdigital electrodes, (c) Deposition of pressure sensitive ink by screen printing.

The screen-printing is done manually using a screen with a mesh size of 180 meshes per centimeter. Electrode geometry, ink layer thickness and, therefore, the overall sensors size can easily be adjusted during the fabrication of the mesh. Excitation and readout of the sensor are simple as only the resistance between the electrodes has to be measured. Figure 3 shows a comparison between the presented sensor (bottom left) and a commercially available FSR from Interlink (top left).

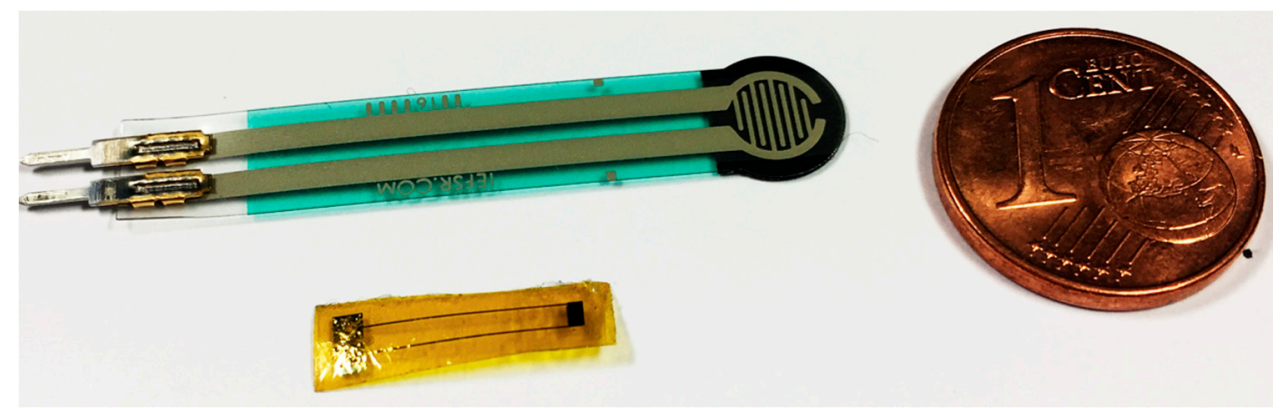

Figure 3. Photography of the presented sensor (bottom left) and a Force Sensing Resistor by Interlink (top left). 
The measurement setups are depicted in Figure 4. The characterization of the sensor has been performed in two ways: First by applying a force with an XYZtec Condor 100 multitesting tool with a $10 \mathrm{~mm}$ by $10 \mathrm{~mm}$ stamp (Figure 4a) and second by applying hydrostatic pressure by compressed air in a pressure chamber (Figure $4 \mathrm{~b}$ ). The maximum force $F$, which has been applied to the sensor in contact pressure measurement, was $40 \mathrm{~N}$. Therefore, the maximum contact pressure was $4 \mathrm{MPa}$. The maximum pressure of compressed air $p$ during testing in the pressure chamber was $0.35 \mathrm{MPa}$.

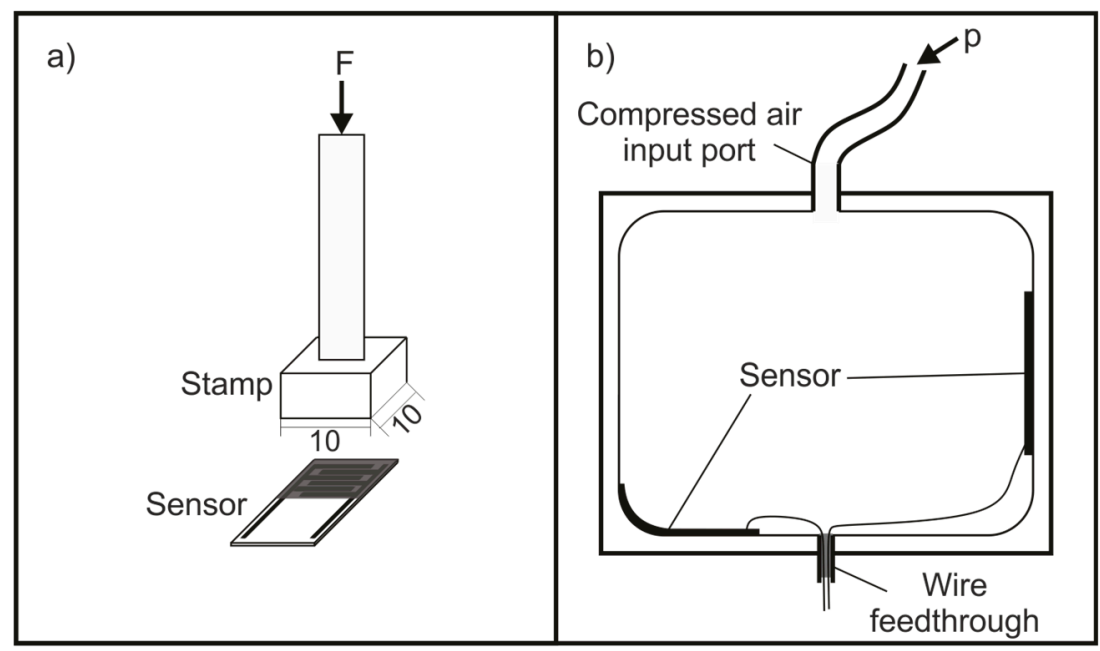

Figure 4. Test setup: (a) Contact pressure measurement (dimensions in mm); (b) Measurement of compressed air pressure in pressure chamber on even and bend surface.

\section{Results}

Sensors with screen-printed silver tracks and a $7 \mu \mathrm{m}$ thick layer of pressure sensitive ink have been tested. The sensors characteristics can be seen in Figure 5. The contact pressure measurement (Figure 5a) shows a sufficient sensitivity up to a pressure of $1.5 \mathrm{MPa}$, going into saturation at higher pressure. The relative change in resistance of the sensors that have been tested inside the pressure chamber (Figure $5 b$ ) is similar to the change during contact pressure measurement up to $0.2 \mathrm{MPa}$ and suddenly decreases strongly. It can be seen, that the application of the sensor on a curved surface does not change the general functionality of the sensor significantly. However, we observed a decrease in the initial resistance from $118.01 \Omega$ to $113.25 \Omega$ due to the bending of the sensor when applied on the curved surface. The change of resistance by bending is not shown in the graph.
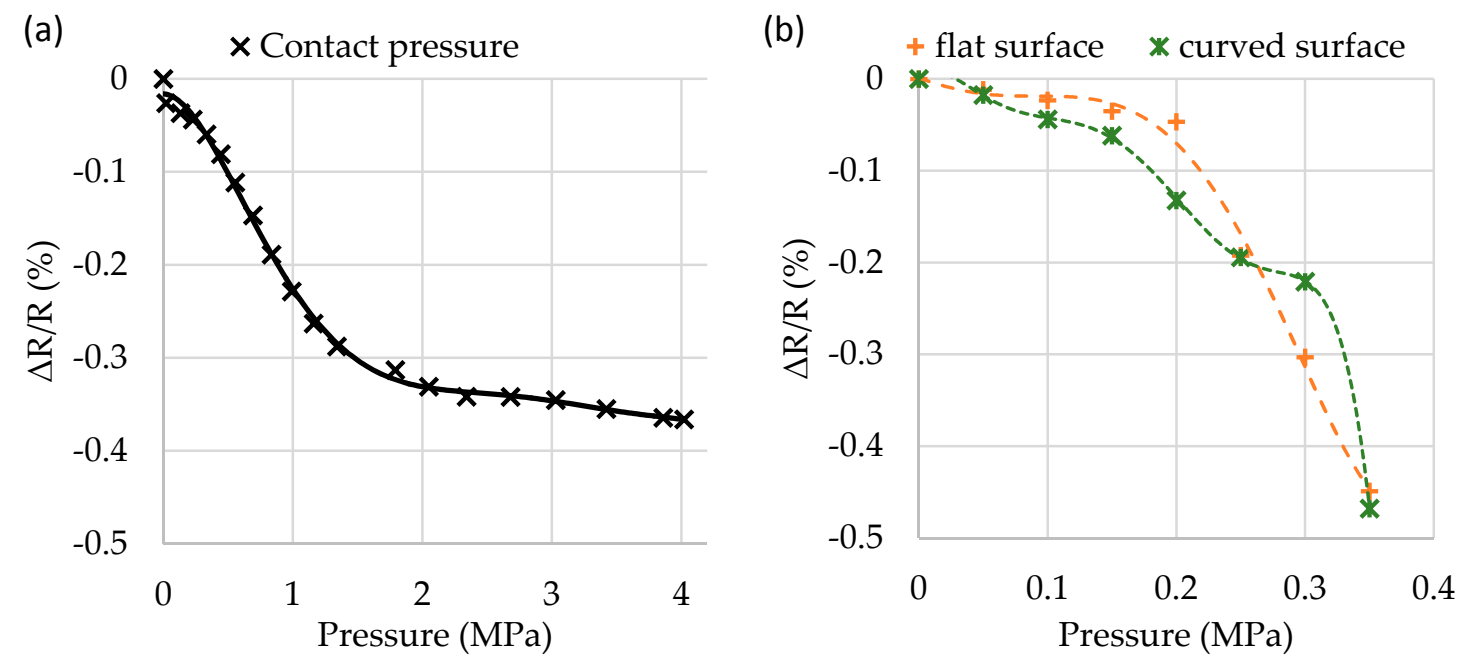

Figure 5. Relative change of resistance vs. applied pressure: (a) Contact pressure measurement; (b) sensor on flat surface (orange dashed line) and sensor on curved surface (green dotted line). 


\section{Conclusions and Outlook}

We have presented a flexible resistive pressure sensor and its fabrication by screen printing. The characterization was done in two different ways. First a contact pressure was applied to the sensor. In a range from 0 to $1.5 \mathrm{MPa}$ the sensor shows nearly linear behavior, while it gets saturated between 1.5 to $4 \mathrm{MPa}$. In a second step, we characterized the sensor in a pressure chamber, applying hydrostatic pressure. In comparison to the contact pressure measurement, a higher sensitivity is achieved. A reasonable explanation for the difference needs further investigations. Finally, the sensor was tested on an even and curved surface at hydrostatic pressure. While the absolute value of the sensor changed due to the bending, the functionality of the sensor did not change significantly. However, the sensitivity of the sensor did change. This is a major problem which need to be solved in the future. Future work will also focus on the characterization of the sensors at higher hydrostatic pressure than $0.35 \mathrm{MPa}$ as well as on the application of the sensor for material integrated pressure sensing.

Acknowledgments: This research was conducted as part of the IGF-project Nr. 18695N from the German Research Association for Measurement, Controls and Systems Engineering supported by the AiF in the funding structure for industrial co-operative research (IGF) by the Federal Ministry of Economics and Technology based on a decision from the German Bundestag.

Conflicts of Interest: The authors declare no conflict of interest. The founding sponsors had no role in the design of the study; in the collection, analyses, or interpretation of data; in the writing of the manuscript, and in the decision to publish the results.

\section{References}

1. Smith, J.H.; Eaton, W.P. Micromachnied Pressure Sensors: Review and Recent Developments. Smart Mater. Struct. 1997, 6, 530-539.

2. Schwerter, M.; Gräbner, D.; Hecht, L.; Vierheller, A.; Leester-Schädel, M.; Dietzel, A. Surface-Passive Pressure Sensor by Femtosecond Laser Glass Structuring for Flip-Chip-in-Foil Integration. J. Microelectromech. Syst. 2016, 25, 517-523.

3. Moghaddam, M.K.; Salas, M.; Ersöz, I.; Michels, I.; Lang, W. Study of resin flow in carbon fiber reinforced polymer composites by means of pressure sensors. J. Compos. Mater. 2017, doi:10.1177/0021998317691877.

4. Fattori, M.; Agostinelli, T.; Pauer, G.; Gold, H.; Cantatore, E. A Fast Printed Pressure and Impact Force Sensing Surface. In Proceedings of the LOPEC International Exhibition and Conference for the printed Electronics Industry, Munich, Germany, 29-30 March 2017.

5. Interlink Electronics, FSR 400 Series Data Sheet. Available online: http://interlinkelectronics.com/datasheets.php (accessed on 28 June 2017).

6. Gerlach, C.; Krumm, D.; Illing, M.; Lange, J.; Kanoun, O.; Odenwald, S.; Hübler, A. Printed MWCNTPDMS-Composite Pressure Sensor System for Plantar Pressure Monitoring in Ulcer Prevention. IEEE Sens. J. 2015, 15, 3647-3656.

7. Huang, X.; Zhi, C. Polymer Nanocomposites: Electrical and Thermal Properties; Springer: New York, NY, USA, 2016.

(C) 2017 by the authors. Licensee MDPI, Basel, Switzerland. This article is an open access article distributed under the terms and conditions of the Creative Commons Attribution (CC BY) license (http://creativecommons.org/licenses/by/4.0/). 